Europhys. Lett., 48 (6), pp. 603-609 (1999)

\title{
A generic input-output analysis of zero-dispersion nonlinear resonance
}

\author{
M.-C. Ho ${ }^{1}$, J.-Y. KO ${ }^{2}$, T.-H. YANG ${ }^{2}$ and J.-L. CHERN ${ }^{2}$ \\ ${ }^{1}$ Department of Physics, National Kaohsiung Normal University \\ Kaohsiung 802, Taiwan, ROC \\ ${ }^{2}$ Nonlinear Science Group, Department of Physics, National Cheng Kung University \\ Tainan 70101, Taiwan, ROC
}

(received 16 August 1999; accepted in final form 15 October 1999)

PACS. 05.45-a - Nonlinear dynamics and nonlinear dynamical systems. PACS. 05.60-k - Transport processes.

\begin{abstract}
When a nonlinear oscillator is driven externally, it may become transparent, i.e., the output response appears without any dispersion or distortion. Such counter-intuitive feature, in fact, is a unique zero-dispersion nonlinear resonance (ZDNR). Herein, it is examined on the base of a generic input-output analysis. The conditions for ZDNR have been clarified and a power law relation has been established to feature the interplay with noise.
\end{abstract}

The study of nonlinear oscillators has become the paradigm of nonlinear dynamics where a variety of new concepts, such as chaos and fractals, has been explored and established [1]. Its impact to physical research is significant since many systems, such as lasers [2], SQUID [3], relativistic oscillators [4], electrical circuits [5], polymeric molecules, and others, can be described by the models of this type. It is also remarkable to notice that nonlinear oscillators can be utilized to describe the dynamics of many physical systems with quantum-mechanical origins. For example, to mention a few, squeezing in classical oscillators [6]. On the other hand, in the classical aspect, resonance is a generic characteristic and the common feature is the dependence of the frequency of an eigenoscillator on its energy in which an extremum exhibits. Surprisingly, in the absence of dissipation, as shown by Soskin [7], a novel type of nonlinear resonance, zero-dispersion nonlinear resonance (ZDNR), can occur when a weak periodic force is applied for which the frequency is close to the external eigenfrequency. Since real systems are subject to dissipation, extension of ZDNR to a dissipative system has been an important issue. As further shown by Luchinsky, McClintock, Soskin, and Mannella, ZDNR can still be developed in dissipative systems [8]. Actually, ZDNR is very unique from a generic input-output point of view. A zero dispersion in spectral domain means that when the phases are fixed, in time domain the output can be exactly the same as the input, which suggests that transparent characteristics have been retrieved for the nonlinear oscillator. A similar feature of transparency also has been found in the interaction between light and matters, e.g., self-induced 
transparency for which short resonant pulses of a given time duration could propagate through a normally absorbing medium with anomalously low attenuation [9]. Thus, zero-dispersion nonlinear resonance can be a classical manifestation of the self-induced transparency in quantum electronics. To illustrate many inherent features of ZDNR as well as its changes in the topology of the basin of attraction, a theory of ZDNR had been developed with an analysis on the action and the phase difference between the force and response [8]. This approach had taken the advantage of the adiabatic process. It neglected the terms of high-frequency oscillation, and thus may be restricted in many practical situations. Still, there remain many fundamental questions on ZDNR, such as to what extent will the driving force be suitable to cast in the ZDNR? Essentially, could ZDNR be a generic property in nonlinear dynamical systems? Should the mismatches in parameters and in sources affect ZDNR critically? Besides, what is the influence of noise on ZDNR? These concerns could be of practical importance and of general interest. In this paper, we employ a generic input-output analysis to clarify these issues.

To illustrate the generality of the input-output approach, we consider a general dynamic system

$$
\ddot{X}+G(X, \dot{X}) \dot{X}+\nabla U=F(t)
$$

in which $X$ is the output, $U$ is the potential, $F(t)$ is the external driving force, and $t$ is the time variable. The overdot is used to denote the time derivate. $G$ is used to include the effects of gain and dissipation. Naively, when the output $X(t)$ is the same as the external driving force $F(t)$, ZDNR can establish. Thus, with a suitable driving force, say $X_{z}$, it may lead to an output $X_{z}$ following $\ddot{X}_{z}+G\left(X_{z}, \dot{X}_{z}\right) \dot{X}_{z}+\nabla U=X_{z}$. The stability of $X_{z}$ can be investigated via a linear stability analysis combined with the Floquet theory $[10,11]$, and one can derive a nonautonomous differential variation equation, i.e., $\delta \ddot{X}+\frac{\partial G}{\partial \dot{X}} \dot{X} \delta \dot{X}+\frac{\partial G}{\partial X} \dot{X} \delta X+G(X, \dot{X}) \delta \dot{X}+$ $\frac{\partial(\nabla U)}{\partial X} \delta X=0$, where $\delta X=X-X_{z}$. This is a generic input-output analysis, and the criteria for ZDNR, thus, could be deduced. However, when there are mismatches in parameters and/or in the forms of driving force, the analysis is complicated and no generic analysis is available. Meanwhile, in real cases, noise is inevitable. The influence of external noise on ZDNR remains to be clarified.

To explore the dynamical features in detail, first we deal with a typical self-excited oscillator in which $G(X, \dot{X})=-\left(a-b \dot{X}^{2}\right)$ and $U=1 / 2 p X^{2}-1 / 4 q X^{4}$. That is

$$
\ddot{X}-\left(a-b \dot{X}^{2}\right) \dot{X}+p X-q X^{3}=F(t),
$$

where the parameters $a$ and $b$ correspond to the gain coefficient and the damping coefficient respectively, $p$ is the coefficient of restoring force in the means of the simple harmonic oscillator, and $q$ is the degree of nonlinearity of the potential. Generally, a time-dependent periodic $F(t)$ will cause $X(t)$ to behave harmonic oscillations, subharmonic oscillations, and even chaos $[1,10]$. To derive the criteria for ZDNR, we set $F(t)=X(t)=X_{z}(t)$, where $X_{z}(t)$ is presumed as the output. But what mathematical form of $F(t)$ will guarantee the generation of ZDNR? Based on the previous discussion, it is clear that if $F_{z}(t)$ satisfy the following equation, then such $F_{z}(t)$ would be the answer:

$$
\ddot{F}_{z}-\left(a-b \dot{F}_{z}^{2}\right) \dot{F}_{z}+(p-1) F_{z}-q F_{z}^{3}=0 .
$$

We illustrate those particular $F_{z}(t)$ of a self-exciting oscillator in fig. 1. By using the method of harmonic balance [11], we derive the approximate solution of limit cycle, $X_{z}(t) \approx A \sin (\omega t)$,

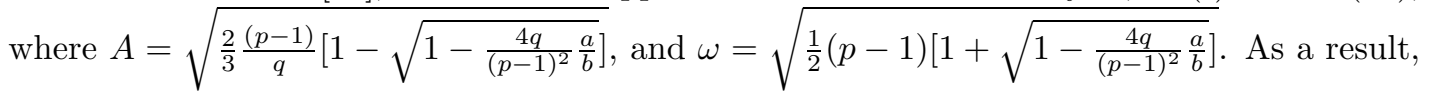



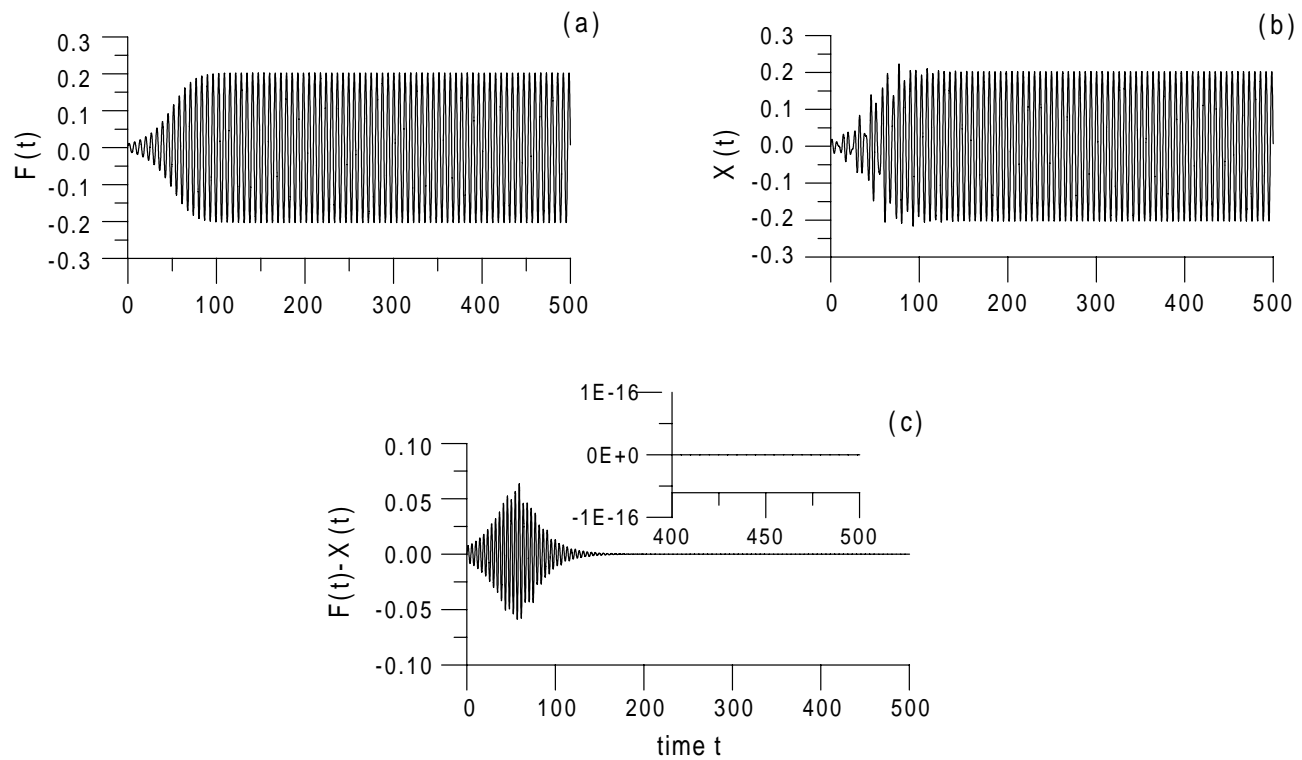

Fig. 1. - The ZDNR phenomenon of a self-excited oscillator.

ZDNR occurs as

$$
\frac{a}{b} \leq \frac{(p-1)^{2}}{4 q}
$$

Direct numerical identification of ZDNR is shown by the dot point in fig. 2. Indeed, ZDNR can occur to a wide range of parameters, though a breakdown of harmonic balance analysis leads to the failure of eq. (4). Here, it is worth noting that the product of $A \omega$ is found to keep as a constant $\sqrt{\frac{4}{3} \frac{a}{b}}$ both in the theoretical analysis and numerical simulation. Actually, the equality $A \omega=\sqrt{\frac{4}{3} \frac{a}{b}}$ is necessary for zero phase difference between $X(t)$ and $F(t)$.

Next, let us explore the effect of external noise on ZDNR of the self-excited oscillator. Without losing generality, we fixed $p=2$ and $q=1$, and the forced self-excited oscillator follows:

$$
\ddot{X}-\left(a-b \dot{X}^{2}\right) \dot{X}+2 X-X^{3}=F(t)+\eta(t),
$$

where $\eta(t)$ is the Gaussian noise satisfying $\langle\eta(t)\rangle=0$ and $\left\langle\eta(t) \eta\left(t^{\prime}\right)\right\rangle=2 D \delta\left(t-t^{\prime}\right)$ in which $D$ is the noise intensity. Here, $\langle\ldots\rangle$ denotes the time average. Naturally, we evaluate the mean-square error $M$ between the output $X(t)$ and the desired ZDNR $X_{Z}$ as

$$
M=\frac{1}{T} \int_{0}^{T}\left[X(t)-X_{z}\right]^{2} \mathrm{~d} t
$$

to quantify the result of the output mismatch. Here the integration time $T$ is taken large enough and $X_{Z}=F(t)=A \sin (\omega t)$ for ZDNR. Since the noise here is one kind of the dynamical noise, the response mismatch should reflect its interplay with the dynamical structure. Hence, a full identification of the interplay requires the inclusion of the noise strength as well as the parameters $a$ and $b$. Nevertheless, one may expect that for a fixed set of $(a, b)$, the output 


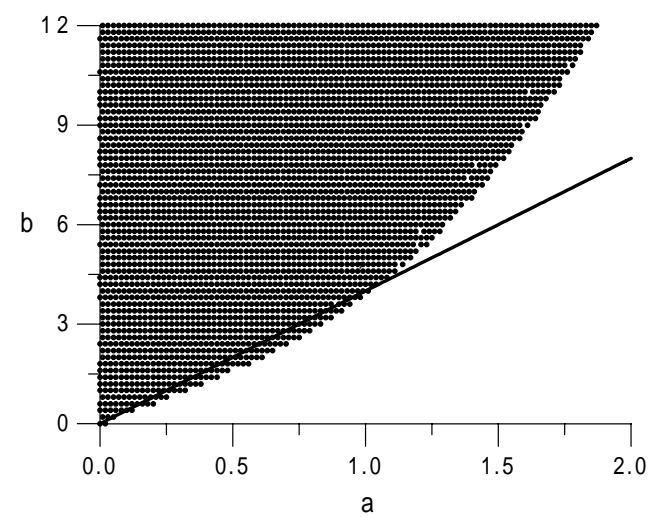

Fig. 2. - The phase diagram of ZDNR in terms of $a$ and $b$. The analytical boundary is shown with the solid line, $\frac{a}{b}=\frac{(p-1)^{2}}{4 q}$, where $p=2$ and $q=1$, and the independent numerical identification of ZDNR is denoted with the dot.

mismatch will vary with the noise strength. As shown by fig. 3(a), the mismatch, here $\sqrt{M}$, is almost linearly proportional to the noise ratio $\sqrt{2 D} / A$ when $a$ and $b$ are fixed. In other words, $\sqrt{M}=\beta \cdot(\sqrt{2 D} / A)$ approximately where $\beta$ is the proportional constant. It is emphasized that each data point in fig. 3(a) is evaluated from 80 different time series and 17000 sampling points (after transience) for each run. We used the Heun algorithm for the integration of eq. (5) [12]. Indeed, the proportional constant $\beta$, exhibited in fig. 3(a), further implies that the interplay between noise and ZDNR can be quantitatively identified. After varying $a$ from 0.1 to 1.1 and $b$ from 0.8 to 70.4 (totally, 320 sets of $a$ and $b$ ), respectively, the dependence of $\beta$ on $a$ and $b$,

(a)

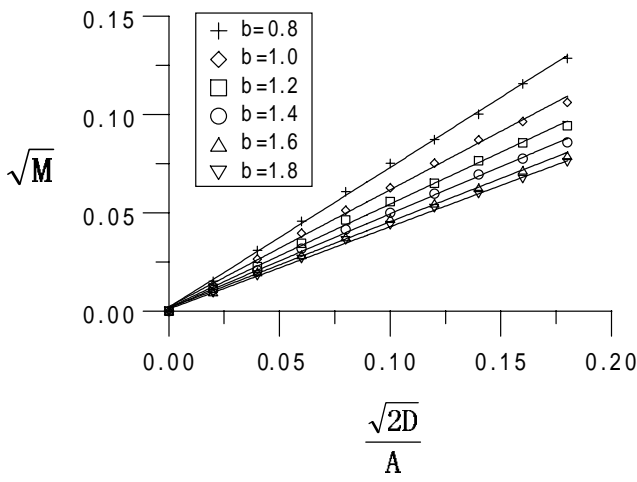

(b)

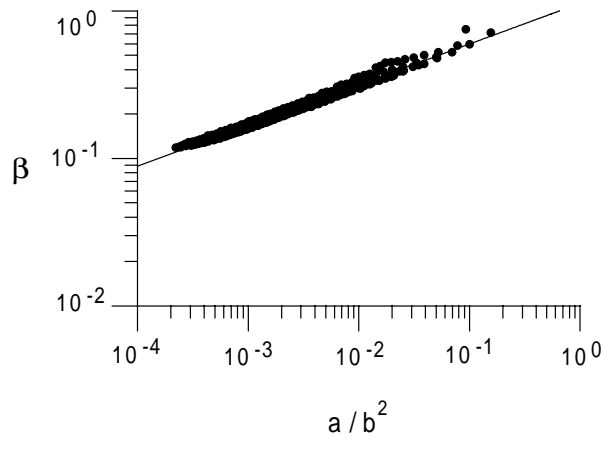

Fig. 3. - a) The output mismatch $\sqrt{M}$ vs. the noise strength $D$ normalized with the amplitude $A$ of ZDNR where $a=0.1$ and the values of $b$ are shown in the legend. The fitting results are shown with the thin lines. b) The power law relation of ZDNR in response to external noise for the self-excited oscillator where $\beta=\sqrt{M} /(\sqrt{2 D} / A)$. The fitting result, also shown by a thin line, follows $\beta=c\left(\frac{a}{b^{2}}\right)^{\alpha}$, where $c=1.13$ and $\alpha=0.276$. Totally, 320 sets of $a$ and $b$ are included. 
as shown in fig. 3(b), can be summarized as

$$
\beta=c \cdot\left(\frac{a}{b^{2}}\right)^{\alpha},
$$

where $c=1.13$ and $\alpha=0.276$. Therefore,

$$
\sqrt{M}=\beta \cdot \frac{\sqrt{2 D}}{A}=c \cdot\left(\frac{a}{b^{2}}\right)^{\alpha} \cdot\left(\frac{\sqrt{2 D}}{A}\right) .
$$

Equation (8) provides the rule for the self-excited oscillator with ZDNR property in response to external noise. The linearity in fig. 3(b) illustrates the powers of influence on mismatch in a clear and easy way for all parameters. It is interesting to note that $\sqrt{M} \propto \sqrt{2 D}$ but $\sqrt{M} \propto\left(a / b^{2}\right)^{\alpha}$, where the exponent $\alpha$ is non-integer. With a fixed mismatch, the suppression of the influence of noise can be achieved by the variation of the dynamical structures, $a$ and $b$. Again, this shows the inherent characteristics of dynamical noise.

Similar schemes demonstrated above can also be applied to the van der Pol oscillator,

$$
\ddot{X}-\mu\left(1-X^{2}\right) \dot{X}+2 X=F(t) .
$$

The detail will be presented elsewhere. In contrast, here we utilized this model to illustrate the sensitivity of ZDNR on source (driving force) mismatch and parameter mismatch. Again, in the case of ZDNR, the dynamics follows: $\ddot{X}_{z}-\mu\left(1-X_{z}^{2}\right) \dot{X}_{z}+2 X_{z}=X_{z}$, where, correspondingly, the driving force is $F_{z}(t)=X_{z}(t)$. Hence, $\ddot{X}_{z}-\mu\left(1-X_{z}^{2}\right) \dot{X}_{z}+X_{z}=0$. By using the method of perturbation, the solution, up to the second order, follows:

$$
X_{z}=\left(2-\frac{1}{8} \mu^{2}\right) \cos \omega t+\frac{3}{4} \mu \sin \omega t+\frac{3}{16} \mu^{2} \cos 3 \omega t-\frac{1}{4} \mu \sin 3 \omega t-\frac{5}{96} \mu^{2} \cos 5 \omega t,
$$

where $\omega=1-\frac{1}{16} \mu^{2}$. We drop the oscillation components of the driving force term by term, starting from the right-hand side of eq. (10) to see the consequence caused by the mismatch in the driving force (source). We again utilize the mean-square errors to quantify the source mismatch, i.e., $\Delta I_{i}=\frac{1}{T} \int_{0}^{T}\left(F_{i}-F_{Z}\right)^{2} \mathrm{~d} t$, where $F_{i}$ means the driving force without the last- $i$ terms, and the output mismatch $M \rightarrow M_{i}$ for the corresponding $i(i=1,2,3,4)$. Simulation shows that all $M_{i} \simeq 2.722 \times 10^{-2}$ as $\Delta I_{1}=9.206 \times 10^{-5}, \Delta I_{2}=8.841 \times 10^{-3}, \Delta I_{3}=$ $8.847 \times 10^{-3}$, and $\Delta I_{4}=2.797 \times 10^{-2}$ for $\mu=0.25$. $M_{i}$ will be reduced when $\mu$ is smaller while, again, $M_{i}$ is roughly a constant for all $\Delta I_{i}$. In contrast, for the case of self-excited oscillator $M \propto \Delta I$. This implies that a small source mismatch does not affect ZDNR seriously. Now, let us further study the influence of parameter $\mu$. When the parameter $\mu$ changes to $\mu+\Delta \mu$, it can be expected that $X(t)$ becomes $X(t)+\Delta X(t)$. Therefore, with eq. (9), it can be shown that approximately

$$
\Delta X \approx\left[-\frac{1}{4} \mu \cos \omega t+\frac{3}{4} \sin \omega t+\frac{3}{8} \mu \cos 3 \omega t-\frac{1}{4} \sin 3 \omega t-\frac{1}{4} \sin 3 \omega t-\frac{5}{48} \mu \cos 5 \omega t\right] \Delta \mu .
$$

After some inequality manipulation, it can be shown that if $\left(\left|\frac{35}{48} \mu\right|+1\right) \mid \Delta \mu \ll 1$, then $|\Delta X| \ll 1$. Indeed, a variation of $\Delta \mu$ still brings out a small $\Delta x$ which suggests the robustness of ZDNR. Particularly, one can verify that similar ZDNR can still be detected even when $\Delta \mu / \mu$ is large. In general, $|\Delta X / \bar{X}|=\alpha|\Delta \mu / \mu|$, where $\bar{X}$ is the maximum of $X$ and $\alpha$ is a small value. This again implies that ZDNR can survive in the cases of parameter mismatch.

In conclusion, we have shown a generic input-output analysis to clarify the zero-dispersion nonlinear resonance in time domain. The condition of ZDNR has been illustrated for the self-excited oscillators as well as for the van der Pol oscillator. We have shown that ZDNR 
can be established even if there are mismatches in control parameter, driving force (source) as well as the inclusion of noise. This is attractive in applications since it implies ZDNR should be a generic property to many physical systems. Practically, an input-output scheme can be utilized to derive the onset conditions and the stability criteria. Furthermore, the influence of noise on ZDNR can be identified where a power law relation exhibits. It is found that the output mismatch $\sqrt{M} \propto \sqrt{2 D}$ for the self-excited oscillator. This is also true for the van der Pol oscillator when $\mu \lesssim 0.05$. Explicitly, in the case of van der Pol oscillator, $\sqrt{M} \propto \sqrt{2 D} \mu$ when $\mu$ is small. When $\mu$ is large, no simple linear correspondence can be deduced. The differences in the responses show that the intrinsic dynamical natures of oscillators are different. On the other hand, it should be addressed that the influence of noise can be dramatically different. Indeed, a significantly contrast feature, i.e., noise-induced linearization, can be achieved in some cases [13]. In such noise-induced linearization cases, the transparency (ZDNR) is mediated by the interplay between the noise and the dynamical structure, while the ZDNR here is inherent to the system's response to the input signal. It should be noted that there remains one factor that we have not fully reported here, i.e., the phase difference between the input and the output. Essentially, an additional relation is required. For example, for the self-excited oscillator described by eq. (2) the ZDNR with a phase difference $\theta$ also needs $\tan \theta=\frac{-a \omega+\frac{3}{4} b \omega^{3} A^{2}}{-\omega^{2}+p-\frac{3}{4} q A^{2}}$. Different relations are required if the systems are changed.

Recently, there are extensive progresses on chaos synchronization and chaos control. The issues of chaos synchronization and chaos control have been extensively investigated in the last few years and a large number of characterization and control schemes has been developed $[10,14]$. In terms of the means of analysis, the calculation of the ZDNR in time domain here is similar to the resonant control method of Hubler and his co-workers [15]. Although the ZDNR in the time domain addressed here can be rephrased as a current question of chaos synchronization and chaos control, i.e., "when does driving lead to a response that coincides with the driven system; or, when do driving and response synchronize even in a noisy environment?", it should be emphasized that the physical concern of ZDNR is different and may be completely irrelevant to chaos. For example, there is no chaos for the model of the self-excited oscillator considered. Indeed, the effects of dispersion and dissipation may be more essential to many nonlinear systems.

$$
* * *
$$

This work is partially supported by the National Science Council, Taiwan, ROC, under project no. NSC87-2112-M-006-010.

\section{REFERENCES}

[1] Lichtenberg A. J. and Libermann M. A., Regular and Chaotic Dynamics (Springer, New York) 1992; Moon F. C., Chaotic and Fractal Dynamics (Wiley, New York) 1992.

[2] Siegman A. E., Lasers (University Science Books, Mill Valley) 1986.

[3] Barone R. and Paterno G., Physics and Applications of the Josephson Effect (Wiley, New York) 1982.

[4] Kim J. H. and Lee H.-W., Phys. Rev. E, 52 (1995) 473.

[5] Hayashi C., Nonlinear Oscillations in Physical Systems (McGraw-Hill, New York) 1964.

[6] Difilippo F., Natarajan V., Boyce K. R. and Pritchard D. E., Phys. Rev. Lett., 68 (1997) 2859; YARIV A., Optical Electronics in Modern Communications, 5th edition (Oxford University Press, New York) 1997.

[7] Soskin S. M., Phys. Rev. E, 44 (1994) R50. 
[8] Luchinsky D. G., McClintock P. V. E., Soskin S. M. and Mannella P., Phys. Rev. Lett., 76 (1996) 4456.

[9] McCall S. L. and Hahn E. L., Phys. Rev. Lett., 18 (1967) 908; Yariv A., Quantum Electronics, 3rd edition (Wiley, New York) 1988.

[10] Nayfeh A. H. and Balachandran B., Applied Nonlinear Dynamics (Wiley, New York) 1995.

[11] Zwillinger D., Handbook of Differential Equations (Academic Press, New York) 1989.

[12] Honerkamp J., Stochastic Dynamical Systems (VCH Publishers, New York) 1994.

[13] Dykman M. I., Luchinsky D. G., Mannella R., McClintock P. V. E., Short H. E., Stein N. D. and Stocks N. G., Phys. Lett. A, 193 (1994) 61; Lih J. S., Ko J.-Y., Chern J.-L., Hsu R.-R., Huang Y.-F. and Ueng H.-Y., Europhys. Lett., 42 (1998) 383.

[14] Otт E., Sauerand T. and Yorke J. A., Coping with Chaos (Wiley, New York) 1994, and references therein.

[15] Hubler A. and Luscher E., Naturwissenschaften, 76 (1989) 67; Georgil R., EberL W., Luscher E. and Hubler A., Helv. Phys. Acta, 62 (1989) 291; Hubler A., Helv. Phys. Acta, 62 (1989) 343. 